\title{
Chip-based squeezing at a telecom wavelength
}

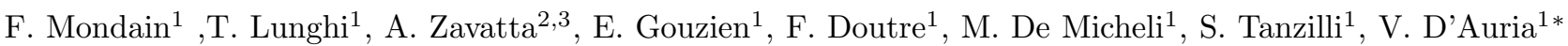 \\ ${ }^{1}$ Université Cote d'Azur, CNRS, Institut de Physique de Nice, Parc Valrose, 06108 Nice Cedex 2, France \\ ${ }^{2}$ Istituto Nazionale di Ottica (INO-CNR) Largo Enrico Fermi 6, 50125 Firenze, Italy \\ ${ }^{3}$ LENS and Department of Physics, Universitá di Firenze, 50019 Sesto Fiorentino, Firenze, Italy
}

\begin{abstract}
We demonstrate a squeezing experiment exploiting the association of integrated optics and telecom technology as key features for compact, stable, and practical continuous variable quantum optics. In our setup, squeezed light is generated by single pass spontaneous parametric down conversion on a lithium niobate photonic circuit and detected by an homodyne detector whose interferometric part is directly integrated on the same platform. The remaining parts of the experiment are implemented using commercial plug-and-play devices based on guided-wave technologies. We measure, for a $\mathrm{CW}$ pump power of $40 \mathrm{~mW}$, a squeezing level of $-2.00 \pm 0.05 \mathrm{~dB}$, (antisqueezing $+2.80 \pm 0.05 \mathrm{~dB}$ ) thus confirming the validity of our approach and opening the way toward miniaturized and easy-to-handle continuous variable based quantum systems.
\end{abstract}

PACS numbers:

\section{INTRODUCTION}

Over the last years, single and multimode squeezed states 11 have played a crucial role in the development of quantum technologies such as quantum computation [2, 3], communication [4] 6], and sensing [7]. In this context, important experimental and theoretical tools for squeezing generation, manipulation, and analysis have been developed [8, 9]. Moreover, it has been proved that squeezed states can be manipulated so as to generate highly non-classical states, such as Schrödinger kittens [10] or cats [1] or hybrid entangled states featuring continuous-discrete variables properties [12, 13].

So far, most of important squeezing demonstrations have been performed by exploiting bulk optics experiments, where squeezed states are usually generated via resonant systems, such as optical parametric oscillators (OPO) [14, 15, and detected thanks to free-space homodyne detections, for which careful spatial alignment and mode matching are required 16. As a consequence, in view of practical applications, we have been assisting to the miniaturisation of important building blocks of squeezing experiments. Compact and stable squeezing generation has been reported in single-pass waveguides [17, 18, and, more recently, in OPO-like devices such as a silicon micro-ring [19] or a waveguide cavity resonator on lithium niobate allowing to measure a squeezing of $-2.9 \mathrm{~dB}$ for a pump power of $\approx 23 \mathrm{~mW} 20$. In parallel, other realisations have reported on-chip homodyne detectors exploiting integrated optics on different substrates [16, 21, 22. Eventually, in the last months, a photonic platform for continuous variable quantum information has been demonstrated on lithium niobate [23.

Our work follows this emerging and very exciting research line. It addresses the realisation of stable, compact, and telecom-compliant squeezing experiments by merging integrated optics on lithium niobate 24 and mature classical technologies. This association allows sat- isfying in a simple way the requirements of easy-to-use and scalable experiments in view of out-of-the-laboratory quantum communication in optical fibres 6, 25. The squeezing generation and the optical coupler for the homodyne detector are fully integrated on a single chip with no significant loss between these two stages and no spatial mode matching concerns [16]. At the same time, with the only exception of homodyne photodiodes, all the other building blocks of our experiment are realised by means of off-the-shelf guided-wave telecom components, fully compatible with existing fibre networks and allowing fast and plug-an-play experiment reconfigurations 26. More in details, squeezed light is generated on-chip via efficient single-pass spontaneous parametric down conversion (SPDC) in a periodically poled waveguide. Squeezing is emitted in the telecom C-band of wavelengths so as to be compatible with low propagation losses in optical fibres. Its homodyne detection, exploiting on-chip optical mixing with the local oscillator (LO) beam, exhibits low losses, essentially due to Fresnel reflection at the chip end-facet and photodiode quantum efficiency. Thanks to this approach, we measure with the integrated homodyne interferometer a raw single-mode squeezing level of $-2.00 \pm 0.05 \mathrm{~dB}(-3.00 \pm 0.05 \mathrm{~dB}$ corrected by avoidable losses), with a continuous wave (CW) pump power of $40 \mathrm{~mW}$. This value validates our approach and represents, to our knowledge, the best squeezing level obtained in miniaturized systems in single-pass $\mathrm{CW}$ pumping regime [17, 20, 23].

\section{EXPERIMENTAL SETUP AND PHOTONIC CHIP}

The experimental setup is sketched in Fig.1. It associates an injection system, exploiting easy-to-assemble guided-wave components, with our home-made lithiumniobate chip. 


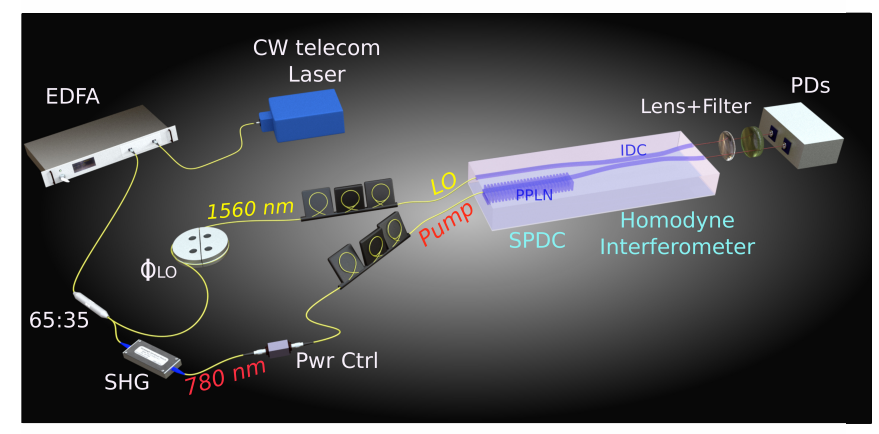

FIG. 1: Experimental setup. A fibre coupled CW telecom laser at $1560.44 \mathrm{~nm}$ is amplified (EDFA) and split into two by means of a 65:35 fibre beam splitter (f-BS). The less intense output (upper arm) serves as local oscillator (LO) while the brighter one (lower arm) is frequency doubled via SHG in a PPLN/RW and used to pump a on-chip squeezing generation stage (SPDC). The power of the beam at $780.22 \mathrm{~nm}$ is controlled with an in-line fibred attenuator (Pwr Ctrl) and its polarisation is adjusted by means of a fibre polarisation controller. At the output of the SPDC stage, squeezed light at $1560.44 \mathrm{~nm}$ is optically mixed with the LO beam inside the same chip in an integrated directional coupler realizing the interferometric part of the homodyne detector. At the chip output, after passing through a bulk lens followed by an optical filter suppressing residual pump at $780.22 \mathrm{~nm}$, light is sent to two InGaAs photodiodes (PDs). The LO phase is scanned thanks to a home-made fibre-stretcher $\left(\phi_{L O}\right)$.

The setup relies on a master, fibre-coupled laser generating a CW optical beam at $1560.44 \mathrm{~nm}$ and amplified up to $0.95 \mathrm{~W}$ with an erbium-doped fibre amplifier (EDFA). At the output of the EDFA, the single mode laser light is split by a high-power 65:35 fibre coupler (f-BS). The less intense output is directed to a homemade fibre stretcher allowing to scan its phase, to be subsequently used as local oscillator for the homodyne interferometer. The brighter output is frequency doubled to $780.22 \mathrm{~nm}$ via second-harmonic generation (SHG) and used to pump the squeezer. The single pass SHG is realised in a commercial periodically poled lithium-niobate ridge waveguide (PPLN/RW [27]) where both input and output ports are fibre-coupled. We note that the ridge output coupling is optimised by the manufacturer so as to maximise the collection only at $780.22 \mathrm{~nm}$. Residual light at $1560.44 \mathrm{~nm}$ at the ridge output is suppressed during its propagation in visible light single mode fibres and by a fibre wavelength demultiplexer (WDM 980/1550, not represented in Fig. 1).

The LO at $1560.44 \mathrm{~nm}$ and the pump at $780.22 \mathrm{~nm}$ are sent to a fibre array and butt-coupled to the home-made photonic chip. A fibre polarisation controller $(\mathrm{PC})$ on each arm is introduced to properly adjust the polarisation. Typical fiber-to-input guide couplings are of $\approx 0.60$. This value can be increased up to 0.92 by inserting taper structures on the photonic chip input [28. The entire optical setup upstream the chip is made using commercial plug-and-play components, exploiting telecom and nonlinear optics technologies and guaranteeing a quick operation with no spatial alignment procedure [16, 26. A schematic of the photonic circuit is presented in Fig.2. The $5 \mathrm{~cm}$-long chip integrates on a single congruent lithium niobate substrate the two key components for squeezing experiments, namely the squeezing generation stage and the optical coupler required for the interferometric part of the homodyne detection. Accordingly, it has two input ports, one for the squeezer pump and the other for the homodyne LO, and two output ports that are directly connected to the bulk homodyne photodiodes. The separation between ports on the same facet is $127 \mu \mathrm{m}$, i.e. compatible with standard fibre-array coupling systems. The waveguide structures are $6 \mu \mathrm{m}$ wide and they are fabricated using soft-proton exchange (SPE) following the technique discussed in Ref. 29]. Propagation losses have been measured to be $\leq 0.04 \mathrm{~dB} / \mathrm{cm}$ at $1560 \mathrm{~nm}$ and $\sim 1 \mathrm{~dB} / \mathrm{cm}$ at $780 \mathrm{~nm}$.

Lithium niobate is particularly suitable to develop integrated squeezers and photonic circuits, featuring high nonlinearity and the possibility of implementing quasiphase-matching to engineer the nonlinear optical response 24. Our on-chip squeezer is a straight periodically poled waveguide, $3 \mathrm{~cm}$ long and designed to have, when pumped at $780.22 \mathrm{~nm}$, a type-0 frequency degenerate SPDC centered at $1560.44 \mathrm{~nm}$. Periodic poling pattern is created by standard electric-field assisted technique with a period $\Lambda=16.3 \mu \mathrm{m}$ [24]. The quasi phasematching temperature for reaching the desired interaction is $104^{\circ} \mathrm{C}$. Such a high temperature has been chosen so as to mitigate light-induced local modifications of refractive index in lithium niobate waveguides. Photorefractive effects are, indeed, particularly evident for optical signals at shorter wavelengths and, at high powers, can induce hopping between different spatial modes [30]. At the chosen working temperature, no mode-hopping is observed for red powers up to $40 \mathrm{~mW}$. Moreover, by exploiting on-chip residual cavity effects and evaluating the resonance positions as functions of the input red 


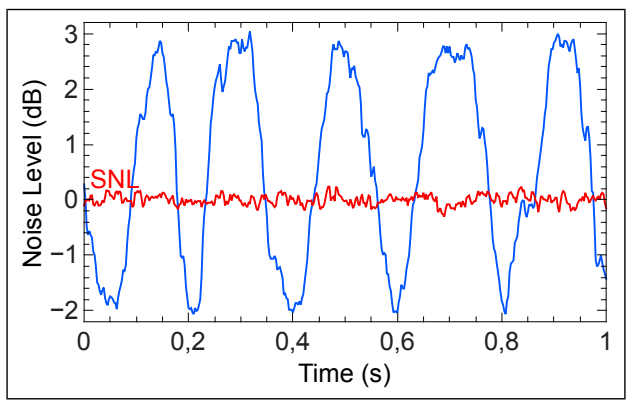

FIG. 2: Schematic of our photonic chip on lithium niobate. The chip includes an SPDC stage, consisting in a periodically poled waveguide $(3 \mathrm{~cm}$ long with poling period $\Lambda=16.3 \mu \mathrm{m})$ for squeezing generation at $1560.44 \mathrm{~nm}$, and an integrated directional coupler realizing the interferometric part of the homodyne squeezing detection. The whole chip length is $5 \mathrm{~cm}$. All waveguide are obtained by soft proton exchange 29] and have a width of $6 \mu \mathrm{m}$. The $127 \mu \mathrm{m}$ spacing between the input (output) waveguides is compatible with of-the-shelf fibrearrays. The homodyne photodiodes are outside the chip and are bulk commercial components.

power, refractive index variations have been measured to be $\leq 4 \times 10^{-5}$. These very small values are consistent with other reported results [31]. The quality of the SPDC stage has been assessed by means of single-photon counting technique. Typical values for our conversion efficiencies are $\sim 10^{5}$ photon pairs $/ \mathrm{mW} / \mathrm{GHz} / \mathrm{s}$ with a FWHM for the spectral emission of $70 \mathrm{~nm}$ 32. We recall that, due to the absence of optical resonator, this value corresponds directly to the squeezing bandwidth [17, 33. The on-chip homodyne interferometer is based on a balanced integrated directional coupler (IDC), exploiting evanescent tail coupling 34. It consists of two waveguides running close to each other over a geometrical length of $\sim 5.5 \mathrm{~mm}$ and with a centre-to-centre separation of $11 \mu \mathrm{m}$. The two IDC inputs are fed with the squeezer output (directly on-chip) and with the local oscillator. Its measured splitting ratio is 50:50 at the SPDC central emission wavelength (i.e. at the LO wavelength). To achieve such a good balancing, the ideal coupling length and BS design have been numerically computed based on our waveguides' typical measured properties. Moreover, in order to comply with fabrication uncertainties, we have realised on the same sample 17 copies of our photonic circuit, corresponding each to a slightly different IDC length, numerically calculated on the basis of our usual parameter fluctuations. The optimal component has been selected among these copies.

The IDC outputs are directed outside the chip and sent to two bulk photodiodes as required for the homodyne detection. The chip is diced using a semiconductor saw and polished at $0^{\circ}$ through chemical mechanical polishing. Due to the absence of anti-reflection coating, light experiences, at its output, a sharp refractive index change at the lithium niobate-air interface and is transmitted with an efficiency $\eta_{F}=1-R_{\text {Fresnel }}$, where $R_{\text {Fresnel }}=\left(\frac{n_{a i r}-n_{\text {chip }}}{n_{a i r}+n_{\text {chip }}}\right)^{2}$. At telecom wavelength, $\eta_{F} \approx 0.86$ [35]. Thanks to a bulk C-coated lens with $11 \mathrm{~mm}$ focal length, the two beams coming out of the photonic circuit are directly imaged on two InGaAs photodiodes (PDs) exhibiting a quantum efficiency $\eta_{P D} \approx$ 0.88 at $1560 \mathrm{~nm}$. By doing so, a nearly perfect chip-tophotodiode coupling is obtained. Residual transmitted light at $780.22 \mathrm{~nm}$ is rejected of more than $-40 \mathrm{~dB}$ with a bulk optical filter, exhibiting a transmission $\eta_{f}=0.99$ at $1560 \mathrm{~nm}$. We stress that the lens, the filter and the homodyne photodiodes are the only bulk optics components in our setup. The difference of the photocurrents from the two photodiodes is amplified by a home-made low-noise trans-impedance amplifier with bandwidth of $\sim 5 \mathrm{MHz}$. Noise power is directly measured with an electronic spectrum analyser set at zero-span centred at $2 \mathrm{MHz}$. For $0.5 \mathrm{~mW} \mathrm{LO}$ power on each photodiode, we obtain an electronic signal-to-noise ratio (SNR) of 12.8 $\mathrm{dB}$. Residual electronic noise effect can be taken into account by introducing an additional loss, through the efficiency $\eta_{e}=(S N R-1) / S N R \approx 0.95$ [36].

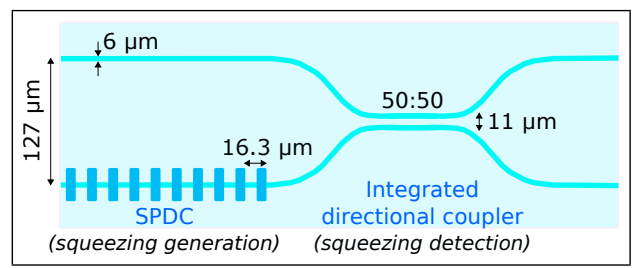

FIG. 3: Normalised noise variances at $2 \mathrm{MHz}$ obtained for a coupled pump power of $40 \mathrm{~mW}$ as a function of the LO phase (proportional to the time) and with a sweep time of $1 \mathrm{~s}$. The electrical spectrum analyser resolution and the video bandwidths are $100 \mathrm{kHz}$ to $30 \mathrm{~Hz}$, respectively.

\section{EXPERIMENTAL RESULTS}

Fig. 3 shows a typical squeezing curve obtained by scanning the phase of the local oscillator over time. We measure a raw squeezing value of $-2.00 \pm 0.05 \mathrm{~dB}$, with an anti squeezing of $2.80 \pm 0.05 \mathrm{~dB}$ when $40 \mathrm{~mW}$ pump light is coupled in the PPLN arm of the chip. The measured squeezed value is affected by losses at the photonic circuit output, such as Fresnel reflection and detectors' non-unit efficiencies as well as electronic noise. However, we underline that these contributions are not due to unavoidable limitations of our chip and can be easily circumvented by means of anti-reflection coating on the chip output facet, leading to $\eta_{F} \approx 1$, and by replacing photodiodes and electronics with more performant ones $\left(\eta_{P D} \cdot \eta_{e} \approx 0.99\right) \quad$ 14. By correcting our measured squeezing for the overall measurement efficiency, $\eta=\eta_{F} \cdot \eta_{f} \cdot \eta_{P D} \cdot \eta_{e}=0.71$, we can infer the squeezing 
at the output of the photonic circuit to be $\sim-3.2 \mathrm{~dB}$ (anti-squeezing of $\sim 3.6 \mathrm{~dB}$ ), which is, to our knowledge, the best reported value for $\mathrm{CW}$-pumped squeezing in waveguides without an optical resonator [17, 20, 23]. We note that the product of squeezing and anti-squeezing net variances is close to the one expected for minimum uncertainty states, thus showing the absence of unwanted excess noise on anti-squeezed quadratures. This value provides evidence of the chip quality in terms of both squeezing generation and losses in the interferometric part of the homodyne detector. We underline that our reported squeezing level is compatible with applications in quantum communication protocols like continuous variable entanglement distribution and teleportation 2 as well as with the heralded generation of Schrödinger cat-like states [10] fully compatible with existing quantum networks and with their use for hybrid entangled states [12, 13. At the same time, we note that the optical chip reported here represents a first important building block for more complex optical circuits able to embrace nonlinear and linear operation stages, enabling new perspectives towards quantum enhanced optical processors [2, 23]. Eventually, we stress that the squeezing levels can be further improved by increasing the SPDC pump powers. In these regimes, to avoid detrimental photo-refractive in the waveguides, an Mg:O-doped lithium niobate substrate should be used for the photonic chip. Based on our previous results this kind of support [26], we expect for a $\mathrm{Mg}$ : $\mathrm{O}$ doped SPE waveguide with the same characteristics as the one reported in this paper, a maximum squeezing from -10 to $-9 \mathrm{~dB}$ for an SPDC pump power $\approx 500 \mathrm{~mW}$.

In conclusion, we have demonstrated a compact and easy-to-handle experiment relying on integrated optics on lithium niobate and off-the-shelf telecom and nonlinear components. Our photonic circuit integrates on-chip the squeezing generation and the homodyne interferometer. The squeezed light generated at telecom wavelength exhibits a raw noise compression of $-2.00 \pm 0.05 \mathrm{~dB}$ for a pump power of $40 \mathrm{~mW}$ with an anti-squeezing of $+2.80 \pm 0.05 \mathrm{~dB}$. The whole remaining setup employs plug-and-play components requiring no alignment procedures for spatial mode matching. These advantages guarantee an extreme reliability and make our approach a valuable candidate for real-world applications based on continuous variable quantum systems.

Funding Information. Agence Nationale de la Recherche (Hy-Light, ANR-17-CE30-0006-01; SPOCQ, ANR-14-CE32-0019), Executive Agency for Higher Education, Research, Development and Innovation Funding (INQCA, PN-II-ID-JRPRO-FR-2014-0013), French government through "Investments for the $\mathrm{Fu}-$ ture" of Université Côte d'Azur UCA-JEDI project (under the label Quantum@UCA, managed by the ANR, ANR-15-IDEX-01). European Regional Development Fund (FEDER) through Project OPTIMAL.

Acknowledgement.A. Z. acknowledges CNR ShortTerm Mobility Program and the Université Nice Sophia Antipolis for invited professor fellowship. Authors thank H. Tronche for technical support.

* Electronic address: virginia.dauria@univ-cotedazur.fr

[1] A. I. Lvovsky, Squeezed light, Photonics Volume 1: Fundamentals of Photonics and Physics, Ed. D. Andrews Wiley, 121-163 (2015).

[2] U. L. Andersen, G. Leuchs, and C. Silberhorn, "Continuous-variable quantum information processing", Laser \& Photon. Rev. 4, 337-354 (2010).

[3] N. C. Menicucci, S. T. Flammia, H. Zaidi, O. Pfister, "Ultracompact generation of continuous-variable cluster states", Phys. Rev. A 76, 010302(R) (2007).

[4] T. Eberle, V. Handchen, J. Duhme, T. Franz, F. Furrer, R. Schnabel and R. F. Werner, "Gaussian entanglement for quantum key distribution from a single-mode squeezing source", New J. Phys. 15, 053049 (2013).

[5] C. Weedbrook, S. Pirandola, R. Garcia-Patron, N. J. Cerf, T. C. Ralph, J. H. Shapiro, and S. Lloyd, "Gaussian quantum information", Rev. Mod. Phys. 84, 621-669 (2012).

[6] M. Huo, J. Qin, J. Cheng, Z. Yan, Z. Qin, X. Su, X. Jia, C. Xie and K. Peng, "Deterministic quantum teleportation through fiber channels", Sci. Adv. 4, eaas9401 (2018).

[7] R. Schnabel, "Squeezed states of light and their applications in laser interferometers", Phys. Rep. 684, 1-52 (2017)

[8] U. L. Andersen, T. Gehring, C. Marquardt, G. Leuchs, "30 years of squeezed light generation", Phys. Scr. 91, 053001 (2016).

[9] A. I. Lvovsky, M. G. Raymer, "Continuous-variable optical quantum-state tomography", Rev. Mod. Phys. 81, 299-332 (2009).

[10] A. Ourjoumtsev, R. Tualle-Brouri, J. Laurat, P. Grangier, "Generating Optical Schrödinger Kittens for Quantum Information Processing", Science 312, 83-86 (2006).

[11] D. V. Sychev, A. E. Ulanov, A. A. Pushkina, M. W. Richards, I. A. Fedorov, A. I. Lvovsky, "Enlargement of optical Schrödinger's cat states", Nat. Photon. 11, 379382 (2017).

[12] A. E. Ulanov, D. Sychev, A. A. Pushkina, I. A. Fedorov, A. I. Lvovsky, "Quantum Teleportation Between Discrete and Continuous Encodings of an Optical Qubit", Phys. Rev. Lett. 118, 160501 (2017)

[13] O. Morin, K. Huang, J. Liu, H. Le Jeannic, C. Fabre and J. Laurat, "Remote creation of hybrid entanglement between particle-like and wave-like optical qubits", Nat. Photon. 8, 570-574 (2014).

[14] M. Mehmet, S. Ast, T. Eberle, S. Steinlechner, H. Vahlbruch, and R. Schnabel, "Squeezed light at $1550 \mathrm{~nm}$ with a quantum noise reduction of $12.3 \mathrm{~dB}$ ", Opt. Express 19, 25763-25772 (2011). 
[15] J. Roslund, R. M. de Araujo, S. Jiang, C. Fabre, and N. Treps, "Wavelength-multiplexed quantum networks with ultrafast frequency combs", Nat. Photon. 8, 109112 (2013).

[16] G. Masada, K. Miyata, A. Politi, T. Hashimoto, J. L. O'Brien and A. Furusawa, "Continuous-variable entanglement on a chip", Nat. Photon. 9, 316-319 (2015).

[17] K. Yoshino, T. Aoki, and A. Furusawa, "Generation of continuous-wave broadband entangled beams using periodically poled lithium niobate waveguides", Appl. Phys. Lett. 90, 041111 (2007).

[18] Y. Eto, T. Tajima, Y. Zhang, and T. Hirano, "Observation of quadrature squeezing in a $\chi^{(2)}$ nonlinear waveguide using a temporally shaped local oscillator pulse", Opt. Express 16, 10650-10657 (2008).

[19] A. Dutt, K. Luke, S. Manipatruni, A. L. Gaeta, P. Nussenzveig, M. Lipson, "On-Chip Optical Squeezing", Phys. Rev. Applied 3, 044005 (2015).

[20] M. Stefszky, R. Ricken, C. Eigner, V. Quiring, H. Herrmann, C. Silberhorn, "Waveguide Cavity Resonator as a Source of Optical Squeezing", Phys. Rev. Applied 7, 044026 (2017).

[21] C. Porto, D. Rusca, S. Cialdi, A. Crespi, R. Osellame, D. Tamascelli, S. Olivares, and M. G. A. Paris, "Detection of squeezed light with glass-integrated technology embedded into a homodyne detector setup", J. Opt. Soc. Am. B 35, 1596-1602 (2018).

[22] F. Raffaelli, G. Ferranti, D. H. Mahler, P. Sibson, J. E. Kennard, A. Santamato, G. Sinclair, D. Bonneau, M. G. Thompson, J. C. F. Matthews, "A homodyne detector integrated onto a photonic chip for measuring quantum states and generating random numbers", Quantum Sci. Technol. 3, 025003 (2018).

[23] F. Lenzini, J. Janousek, O. Thearle, M. Villa, B. Haylock, S. Kasture, L. Cui, H.-P. Phan, D. Viet Dao, H. Yonezawa, P. K. Lam, E. H. Huntington, M. Lobino, "Integrated photonic platform for quantum information with continuous variables", Sci. Adv. 4, eaat9331 (2018).

[24] O. Alibart, V. D'Auria, M. D. Micheli, F. Doutre, F. Kaiser, L. Labonté, T. Lunghi, E. Picholle, S. Tanzilli, "Quantum photonics at telecom wavelengths based on lithium niobate waveguides", Journ. Opt. 18, 104001 (2016).

[25] F. Y. Hou, L. Yu, X. J. Jiaa, Y. H. Zheng, C. D. Xie, and K. C. Peng, "Experimental generation of optical non- classical states of light with $1.34 \mu \mathrm{m}$ wavelength", Eur. Phys. J. D 62, 433-437 (2011).

[26] F. Kaiser, B. Fedrici, A. Zavatta, V. D'Auria, and S. Tanzilli, "A fully guided-wave squeezing experiment for fiber quantum networks", Optica 3, 362-365 (2016).

[27] T. Umeki, O. Tadanaga, and M. Asobe, "Highly Efficient Wavelength Converter Using Direct-Bonded PPZnLN Ridge Waveguide", IEEE J. Quantum Electron. 46, 1206-1213 (2010).

[28] D. Castaldini, P. Bassi, S. Tascu, P. Aschieri, M. P. De Micheli, and P. Baldi, "Soft-Proton-Exchange Tapers for Low Insertion-Loss LiNbO3 Devices", J. Light. Technol., 25, 1588-1593 (2007).

[29] L. Chanvillard, P. Aschieri, P. Baldi, D. B. Ostrowsky, M. de Micheli, L. Huang, D. J. Bamford, "Soft proton exchange on periodically poled LiNbO3: A simple waveguide fabrication process for highly efficient nonlinear interactions", App. Phys. Lett. 76, 1089-1091 (2000).

[30] A. M. Glass, D. von der Linde, D. H. Auston, and T. J. Negran, "Excited state polarization, bulk photovoltaic effect and the photorefractive effect in electrically polarized media", J. Electron. Mater. 4, 915-943 (1975).

[31] A. Hellwig, Nonlinear optical and photorefractive properties of periodically poled channel waveguides in lithium niobate, Ph.D. thesis, Universitat Paderborn (2011).

[32] L. A. Ngah, O. Alibart, L. Labonté, V. D'Auria, S. Tanzilli, "Ultra-fast heralded single photon source based on telecom technology", Laser \& Photon. Rev. 9, L1-L5 (2015).

[33] M. Pysher, R. Bloomer, C. M. Kaleva, T. D. Roberts, P. Battle, O. Pfister, "Broadband amplitude squeezing in a periodically poled KTiOPO4 waveguide", Opt. Lett., 34, 256-258 (2009).

[34] D. Barral, M. G. Thompson, J. Linares, "Detection of two-mode spatial quantum states of light by electro-optic integrated directional couplers", Journ. Opt. Soc. Am. B 32, 1165-1173 (2015).

[35] D. E. Zelmon, D. L. Small, D. Jundt, Journ. " Infrared corrected Sellmeier coefficients for congruently grown lithium niobate and 5 mol.\% magnesium oxide-doped lithium niobate", Opt. Soc. Am. B 14, 3319-3322 (1997).

[36] J. Appel, D. Hoffman, E. Figueroa, and A. I. Lvovsky, "Electronic noise in optical homodyne tomography", Phys. Rev. A 75, 035802 (2007). 